\title{
Side Branch is the Main Determinant Factor of Bifurcation Lesion Complexity: Critical Review with a Proposal Based on Single-centre Experience
}

Imad Sheiban, ${ }^{1}$ Filippo Figini, ${ }^{1}$ Valeria Gasparetto, ${ }^{1}$ Fabrizio D'Ascenzo, ${ }^{2}$ Claudio Moretti ${ }^{3}$ and Filippo Leonardo ${ }^{1}$

1. Ospedale "Pederzoli", Peschiera del Garda, Italy; 2. Città della Salute e della Scienza Hospital, University of Turin, Turin, Italy;

3. Ospedale di Chivasso, Turin, Italy

DOI: https://doi.org/10.17925/HI.2021.15.2.67

A though bifurcation stenting can be often managed with a simple provisional approach, in some settings, more complex techniques are appropriate. Based on our clinical experience and on data from literature, we propose a simple algorithm that may assist in selecting cases for elective double stenting. We found that, when the side branch is of adequate dimensions and affected by significant disease (longer than $10 \mathrm{~mm}$ and/or with presence of ostial calcifications), double stenting is associated with a lower incidence of adverse events, compared with provisional stenting.

\section{Keywords}

Percutaneous coronary intervention, bifurcation lesions, elective double stenting, stent

Disclosures: Imad Sheiban, Filippo Figini, valeria Gasparetto, Fabrizio D'Ascenzo, Claudio Moretti and Filippo Leonardo have no financial or non-financial relationships or activities to declare in relation to this article.

Review process: Double-blind peer review.

Compliance with ethics: All procedures were followed in accordance with the responsible committee on human experimentation and with the Helsinki

Declaration of 1975 and subsequent revisions. This article involves a review of literature, and also a discussion of retrospective clinical data. Informed consent was received for all patients involved, as well as agreement for the scientific use of their anonymized personal data. Data availability: The datasets generated during and/or analysed during the current study are available from the corresponding author on reasonable request.

Authorship: The named authors meet the International Committee of Medical Journal Editors (ICMJE) criteria for authorship of this manuscript, take responsibility for the integrity of the work as a whole, and have given final approval for the version to be published.

Access: This article is freely accessible at touchCARDIO.com. (c) Touch Medical Media 2021.

Received: 11 October 2021

Accepted: 8 December 2021

Published online: 21 December 2021

Citation: Heart International. 2021;15(2):67-72

Corresponding author: Filippo Figini, Ospedale Pederzoli, via Monte Baldo 24, Peschiera del Garda, 37019, VR, Italy. E: ffigini@ospedalepederzoli.it

Support: No funding was received in

the publication of this article.
Available evidence from randomized studies suggests that most bifurcation lesions are best managed with a provisional stenting (PS) approach, as this is associated with fewer complications, simpler procedures and better outcomes, compared with elective double stenting. ${ }^{1-6}$ However, the Achilles' heel of single-stent strategies is represented by a higher risk of suboptimal result on the side branch, either acutely or during follow-up. ${ }^{6}$ Moreover, complex bifurcations were under-represented in randomized trials, which mainly included lesions that the operators considered potentially suitable for a single-stent approach.7.8

When planning bifurcation stenting, it is advisable to consider variables that influence a) the probability of an adverse event on the side branch, and b) its clinical consequences. The risk of side-branch compromise or restenosis is largely determined by the extent of disease, while its clinical significance is associated with the quantity of myocardium it supplies. If the side branch is large, but not significantly affected by atherosclerosis, PS is generally recommended; the same holds true for a severely stenotic side vessel that has a minor territory of distribution.?

It is generally accepted, however, that extensive disease involving a clinically significant side branch might be best treated using a double-stenting approach upfront, provided that it is performed with optimal technique: as it is often said, the strategy is dictated by the side branch. The problem, however, is that the concepts of 'clinical significance' and 'disease complexity' are not universally defined.? In the present paper, we will summarize currently available definitions and propose a standardized approach.

\section{Single-centre experience: methods and results}

We retrospectively reviewed consecutive patients treated with percutaneous coronary intervention (PCl) on a bifurcation lesion at our centre from 2010 to 2012. Only lesions with a clinically relevant side branch (diameter $\geq 2.5 \mathrm{~mm}$, length $>75 \mathrm{~mm}$ ) were taken into account; vessel size was assessed visually. The decision to implant one or two stents as intention-to-treat, as well as the procedural aspects, were decided by the operator. Patients undergoing PS (as intention-to-treat) who required bailout side-branch stenting were excluded from the study.

Based on data from the literature, we defined 'complex bifurcations' as lesions with the following features: true bifurcations with side branch disease extending for more than $10 \mathrm{~mm}$; Medina class 1,1,1 and calcifications at side branch ostium. Other cases were categorized as 'simple bifurcation lesions'. 
Table 1: Demographics and clinical characteristics of patients with simple and complex bifurcation lesions

\begin{tabular}{|c|c|c|c|}
\hline & $\begin{array}{l}\text { Simple lesions } \\
(n=248 ; 70 \%)\end{array}$ & $\begin{array}{l}\text { Complex lesions } \\
(n=106 ; 30 \%)\end{array}$ & $\mathrm{p}$ value \\
\hline Age, mean $\pm S D$, years & $67.4 \pm 5.6$ & $69.4 \pm 3.4$ & 0.45 \\
\hline Female gender, $\mathrm{n}(\%)$ & $92(37.4)$ & $36(33.9)$ & 0.54 \\
\hline Hypertension, n (\%) & $197(79.4)$ & $87(82.4)$ & 0.45 \\
\hline Hyperlipidaemia, n (\%) & $179(72.3)$ & $66(62.5)$ & 0.21 \\
\hline Diabetes mellitus, n (\%) & $69(28.1)$ & $32(30.1)$ & 0.42 \\
\hline Previous myocardial infarction, n (\%) & $86(34.5)$ & $42(39.4)$ & 0.67 \\
\hline Smoking habit, $n$ (\%) & $94(37.3)$ & $36(34.7)$ & 0.41 \\
\hline $\begin{array}{l}\text { Acute coronary syndrome, n (\%) } \\
\text { - Unstable angina } \\
\text { - NSTEMI } \\
\text { - STEMI }\end{array}$ & $\begin{array}{l}92(37.3) \\
32(12.8) \\
52(21.1)\end{array}$ & $\begin{array}{l}35(33.3) \\
15(13.9) \\
15(13.9)\end{array}$ & 0.12 \\
\hline
\end{tabular}

NSTEMI = non-ST-elevation myocardial infarction; SD = standard deviation; STEMI = ST-elevation myocardial infarction.

Figure 1: Three-year outcomes for the overall population (panel A) and for the subgroup with distal left main disease (panel B)

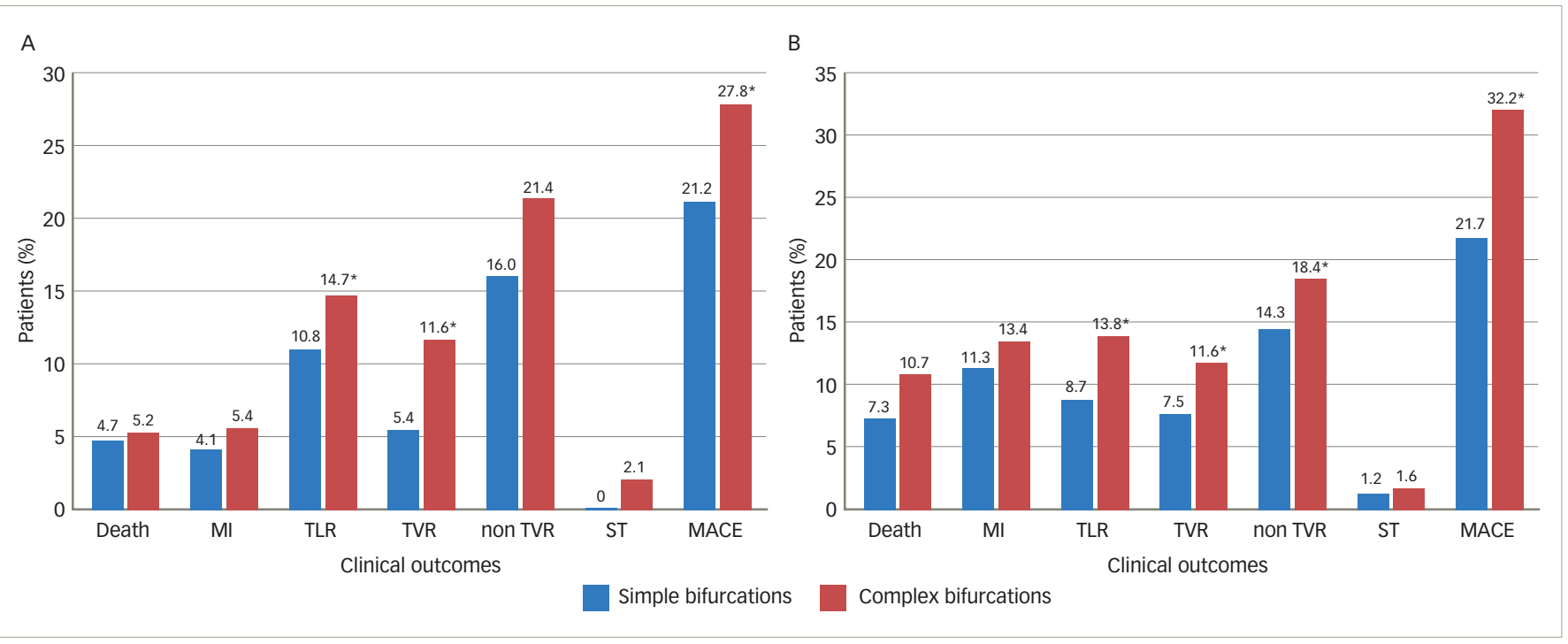

${ }^{*} p<0.05$

MACE = major adverse cardiovascular events; $M I=$ myocardial infarction; $S T=$ stent thrombosis; $T L R=$ target-lesion revascularization; $T$ VR $=$ target-vessel revascularization.

All patients included had a complete 3-year clinical follow-up, and rates of death, myocardial infarction, target vessel revascularization (TVR), target lesion revascularization (TLR), stent thrombosis and major adverse cardiovascular events (MACE - defined as a composite of death, myocardial infarction and TVR) were recorded.

A total of 354 patients were included in this analysis; of these, $248(70 \%)$ had a simple bifurcation and 106 (30\%) had a complex bifurcation lesion. Baseline demographics and clinical characteristics were not significantly different between patients with simple and complex bifurcations (Table 1). Distal left main disease was present in 68 patients (19\%). Side-branch lesions were significantly longer in the complex group than the simple group $(18.0 \pm 4.2 \mathrm{~mm}$ versus $5.7 \pm 2.8 \mathrm{~mm}$, respectively). Accordingly, an elective double-stenting technique was predominantly used in this subset (83\% of complex group versus $28 \%$ of simple group; $p<0.0001$ ), mainly with modified $T$ stenting or the minicrush technique.

The rate of MACE at follow-up was significantly higher in patients with complex lesions compared with simple lesions (27.8\% versus $21.2 \%$, respectively; $p<0.05)$ mainly due to higher incidence of TVR. The same was true in patients with distal left main disease (Figure $1 A, B$ ). In patients with Medina 1,1,1 lesions, side-branch disease $>10 \mathrm{~mm}$ and side-branch calcification, incidence of MACE at 3 years reached $37.8 \%$. The rate of death, myocardial infarction and stent thrombosis was numerically higher in patients with complex bifurcations, although these comparisons did not reach statistical significance.

If we consider our definition of 'complex bifurcation lesion' as an indication for elective double stenting, the operator's decision was in agreement with lesion definition (i.e. PS in simple lesions, double stenting in complex cases) in 267 patients (75\%).

When only subjects with simple lesions were considered, the rate of MACE at follow-up was significantly higher in patients treated with two stents compared with PS (26.9\% versus $21.2 \%$, respectively; $p<0.01)$. Conversely, the rates of MACE, TLR and TVR was markedly increased in patients with complex bifurcations treated with PS compared with elective double stenting (MACE $52.2 \%$ versus $22.4 \%$, respectively; $\mathrm{p}<0.01$; Figure $2 A, B)$. 
Figure 2: Clinical outcomes according to treatment strategy in patients with simple bifurcations (panel A) and complex bifurcation lesions (panel B)

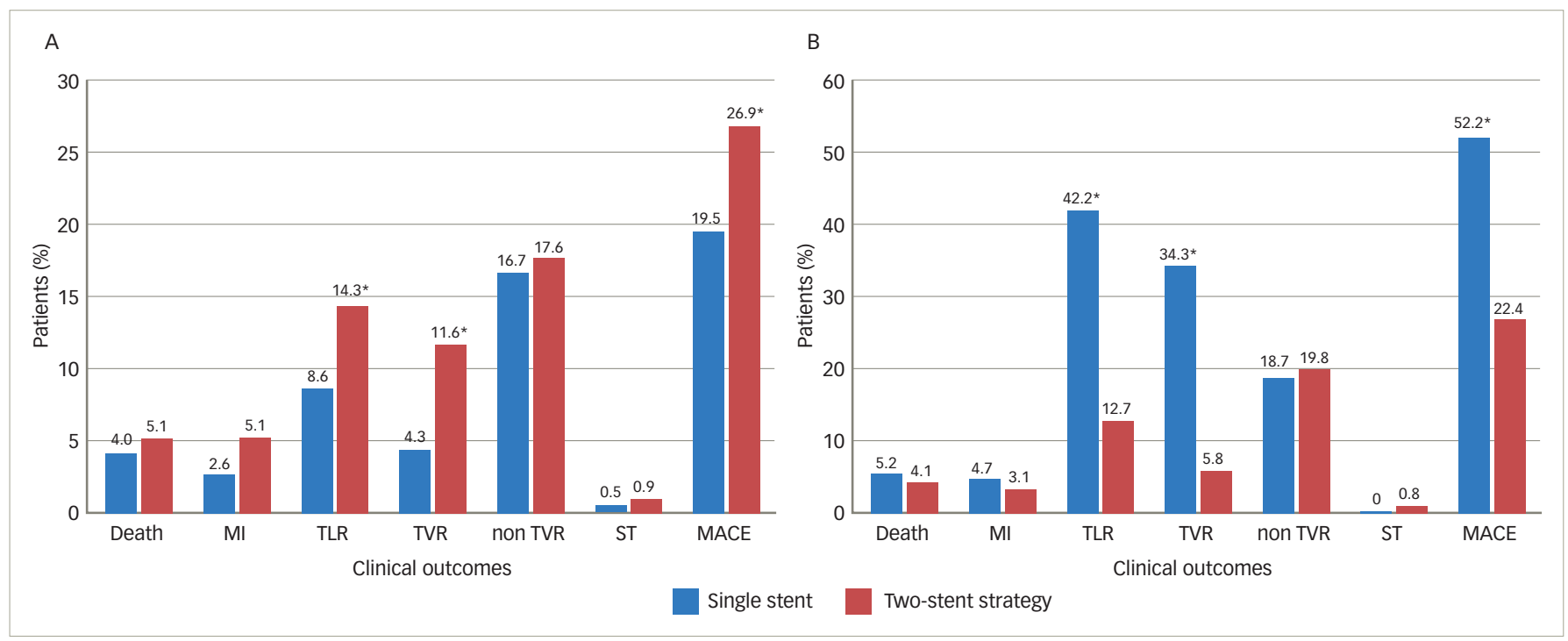

${ }^{*} p<0.05$

MACE = major adverse cardiovascular events; $M I=$ myocardial infarction; ST = stent thrombosis; $T$ LR = target-lesion revascularization; TVR = target-vessel revascularization.

\section{Discussion}

This analysis shows the proposed definition predicted the risk of subsequent adverse events in patients undergoing $\mathrm{PCl}$ for bifurcation lesions. Importantly, in situations classified as simple, a provisional approach was associated with better outcomes, while in the complex anatomy subgroup, the risk of adverse events improved with an elective two-stents strategy. In this analysis, the exclusion of patients undergoing bailout side-branch stenting after provisional technique probably underestimates the benefit of double stenting as intention-to-treat in complex cases.

Our results are in accordance with data reported in the Definitions and impact of complex bifurcation lesions on clinical outcomes after $\mathrm{PCl}$ using drug-eluting stents (DEFINITION) I study, where patients with complex bifurcations (defined according to the trial's criteria) had lower 1-year mortality when treated with the two-stents technique. ${ }^{9}$ This concept is being prospectively tested in the randomized DEFINITION II trial, which only includes patients with complex bifurcations, as defined by the DEFINITION I study criteria. ${ }^{10}$ Our trial confirmed that, in complex bifurcation, double elective stenting significantly improves the clinical outcomes compared with PS.

The Medina classification, despite its great use in standardizing definitions, is not sufficient to discriminate between lesions that benefit from elective double stenting and those that do not. Despite extensive research in this field, the optimal treatment strategy in many bifurcation lesions is still a matter of debate. Many studies in this area have two major pitfalls. Firstly, due to selection bias, complex bifurcations have been under-represented. Secondly, their main goal often was to identify the best single-stenting strategy for most situations; however, it is clear that, given the wide anatomical variety of these lesions, treatment should be tailored to every specific situation.

In our opinion, universal definitions of simple and complex bifurcation, which should be adopted for selecting stenting strategy, are still lacking. An algorithm mainly based on angio-anatomical characteristics of bifurcation lesions can represent the most appropriate solution, since it is simple, can be done online during the procedure and can guide the operator in selecting the most appropriate strategy (one versus two stents) for every situation.

The correct approach is generally dictated by the side branch: in cases where this is clinically relevant and extensively diseased, a double-stenting technique should be pursued as intention-to-treat. The best and more simple approximation for clinical relevance is vessel size $(\geq 2.5 \mathrm{~mm})$ and length $(75 \mathrm{~mm})$. Yet PS still is preferable for a significant side branch, provided that it has no or minimal disease, or stenosis is severe but short ( $<10 \mathrm{~mm}$ in length). Our data suggest that double stenting in this context has a price to pay in terms of TVR at follow-up.

\section{Clinical relevance of the side branch}

Generally, the authors find that the diameter of the side branch is directly proportional to the mass of myocardium that it supplies; as a rule of thumb, any side branch $\geq 2.5 \mathrm{~mm}$ is considered physiologically relevant. There are, of course, other variables to be taken into account. For example, in the authors' experience, vessel diameter overestimates its functional significance if a portion of myocardium it supplies is not viable, or when the artery is ectatic because of wall disease; on the contrary, a small branch can be clinically important if it provides collateral circulation to an occluded artery, or if its disease is associated with significant symptoms.

Most available literature on nuclear stress imaging has adopted a threshold of $10 \%$ of ischaemic myocardium to define moderate-severe ischaemia; such a finding is indeed associated with a yearly risk of myocardial infarction or coronary death of around 5\%." Based on these data, it is important to obtain an optimal revascularization on any vessel supplying at least $10 \%$ of myocardial mass. Indeed, 10-13\% of ischaemic myocardium is the threshold over which the survival benefit of $\mathrm{PCl}$ over medical therapy becomes apparent; ;2 above this level, the prognostic benefit of PCl increases with the amount of inducible ischaemia. From the authors' personal observations, the problem when dealing with bifurcation lesions is that, even if a pre-procedural single-photon emission computed tomography (CT) scan is available, we cannot precisely estimate the fraction of ischaemic myocardium attributable to the side branch. 
Coronary CT angiography allows us to calculate the fractional myocardial mass (FMM) supplied by each vessel. Kim et al. have demonstrated that, excluding the left main (where the side branch almost always provides blood to a significant amount of myocardium), only one in five bifurcations involves a side branch with a FMM greater than $10 \%$. Side-branch length $\geq 73 \mathrm{~mm}$ (measured at CT scan) identifies FMM $\geq 10 \%$ with a sensitivity and specificity of around $80 \% .^{13}$ These results are interesting because, in the future, it is expected that more patients will undergo coronary catheterization after a CT scan, which can be used to programme revascularization strategy upfront. ${ }^{14}$ In the study by Kim et al., vessel diameter was not significantly associated with FMM, and most side branches did not supply a significant amount of myocardium, even if their diameter was only slightly smaller than the main vessel; accordingly, FFR values were higher in the side branch despite a similar degree of stenosis to the main vessel. ${ }^{13}$

In order to better estimate the clinical significance of a diagonal branch, a scoring system has been proposed incorporating vessel diameter, and number and distribution of diagonal branches; however, angiographic parameters could not reliably predict the functional significance of diagonal branches..$^{15}$

Fractional flow reserve (FFR) can provide useful information regarding the significance of side-branch disease. However, several caveats must be kept in mind:

- Even if FFR is influenced by the territory of distribution of the target vessel, a positive FFR does not automatically imply that the side branch is clinically significant. ${ }^{13}$

- Pre-intervention FFR results for the side branch do not correlate with post-procedural FFR; this method is therefore of little help in deciding upfront which lesions deserve an elective double stenting. It is more useful when determining the need for a further stent on the side branch after PS. ${ }^{16}$

- Patients with no significant ischaemia after the procedure remain at risk of subsequent side-branch restenosis. ${ }^{16}$

From a technical viewpoint, it can sometimes be difficult to recross the side branch with the pressure wire; however, the wire can be safely jailed, still providing accurate measurements. ${ }^{17,18}$

A recent study has confirmed the prognostic value of FFR in patients treated with a single stent on the distal left main to left anterior descending artery. Outcomes at 5 years were better if FFR was $>0.80$ on the left circumflex artery; interestingly, there was no significant correlation between FFR and percent stenosis. ${ }^{19}$ This is in agreement with results from Koo et al. who showed that, after single stenting, most ostial side-branch lesions that appear critical at visual estimation are not significant at FFR evaluation. ${ }^{20}$ Theoretically, acute FFR levels could be influenced by periprocedural oedema and thrombus, but follow-up studies have shown that FFR tends to remain stable over time..$^{16,21}$

On the other hand, the DKCRUSH-VI study (Randomized study on DK crush technique versus provisional stenting technique for coronary artery bifurcation lesions; ChiCTR-TRC-07000015) compared FFR-guided with an angio-guided strategy for bailout side-branch stenting after stent implantation on the main vessel..$^{22}$ In the two groups, the side branch was stented if FFR was $<0.80$ (in FFR group), or in presence of Thrombolysis in Myocardial Infarction (TIMI) flow grade $<3$, stenosis $>70 \%$ or dissection type $>$ A (in the angiography group). The FFR-guided approach resulted in a trend for less side-branch stenting, with no significant reduction in MACE at 1 year.22

\section{Risk of side-branch compromise}

Kang et al. showed that pre-intervention minimal lumen area and plaque burden at side branch ostium - measured by intravascular ultrasound (IVUS) - were associated with FFR $<0.80$ after main-vessel stenting; a cut-off of $2.4 \mathrm{~mm}^{2}$ and $51 \%$, respectively, were identified. ${ }^{23}$ However, they showed that only half of side branches having both these features ended up in haemodynamically significant stenosis after main-vessel stenting. The authors identified no reliable pre-intervention IVUS predictor of subsequent side-branch compromise. ${ }^{23}$ Post-procedural IVUS parameters also were not accurate in predicting functional side-branch obstruction. While nearly half of side branch ostia had a post-procedural area stenosis $>50 \%$, only $15 \%$ were functionally significant; plaque shift superimposed on carina shift was necessary to cause significant obstruction. ${ }^{24}$

In the Korean coronary bifurcation stenting registry (COBIS I; ClinicalTrials.gov identifier: NCT01642992), pre-procedural side-branch stenosis $>50 \%$, lesion length on side branch, proximal main-vessel stenosis $>50 \%$ and acute coronary syndrome were identified as predictors of side-branch occlusion after main-vessel stenting. ${ }^{25}$

Dou et al. proposed the RESOLVE score, which includes plaque distribution (on the opposite or same side as the side branch), pre-procedural TIMI flow grade, bifurcation angle, diameter ratio between the main vessel and side branch, stenosis degree at the level of bifurcation core and side branch. The risk of side-branch occlusion ranged from $0 \%$ in the lower quartile of RESOLVE scores to nearly $20 \%$ in the higher quartile. ${ }^{26}$

In clinical practice, when selecting appropriate strategy, most operators focus on lesion length of the side branch using a threshold of $>5 \mathrm{~mm}$ or $>10 \mathrm{~mm}$ to consider elective double stenting. Indeed, a long stenosis will have a high risk of restenosis if treated with balloon angioplasty only; moreover, placement of a long stent on the side branch will be technically less favourable after main-vessel stenting, thereby suggesting a 'side branch first' approach. ${ }^{27}$ A recent meta-analysis confirmed that bifurcation lesions with side-branch stenosis $>10 \mathrm{~mm}$ had better results when treated with two-stent techniques. ${ }^{27}$

The role of bifurcation angle is somewhat controversial, but some studies have found a higher risk of side-branch occlusion with a wide angle. ${ }^{26,28,29}$ A T-shaped anatomy also increases technical difficulty of recrossing, and could prompt clinicians to stent the side branch first with a T-stent technique rather than culotte or crush. ${ }^{28}$

Moderate or severe calcifications can be identified in around $20 \%$ of bifurcation lesions, and is associated with worse outcomes, mainly because of target-lesion and vessel failure. ${ }^{30,31}$ In our experience, in case of acute side-branch occlusion, calcification at its ostium often makes rewiring and rescue more difficult. Indeed, the difficulty in side-branch wiring should be taken into account before proceeding to main-vessel stenting: if the side branch is clinically relevant, significantly diseased and difficult to wire, it is strongly advisable to secure it by stenting upfront. Accurate lesion preparation, including rotational atherectomy, may be advisable in the case of severe calcification. ${ }^{32-34}$

\section{Proposed algorithm}

While PS often provides excellent results with less effort, when bailout side-branch stenting is necessary, the rate of adverse events increases steadily, compared with double stenting. ${ }^{35}$ Some data suggest that results after elective double stenting are better than for cases treated with bailout side-branch stenting in the context of a provisional technique. ${ }^{35}$ 


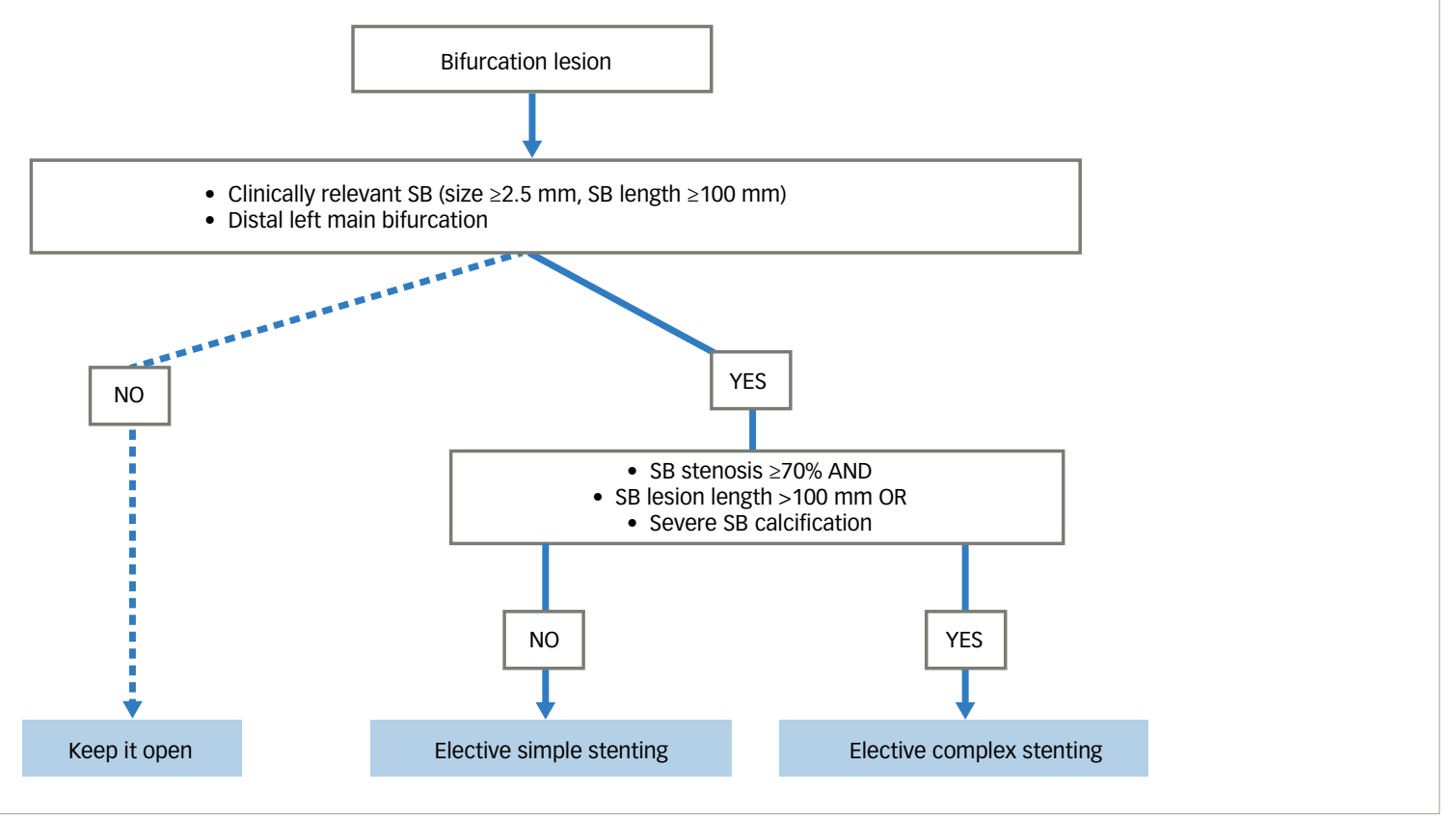

$S B=$ side branch

Therefore, if we could reliably predict the risk of side-branch compromise upfront, it would be preferable to routinely perform a two-stent strategy in those cases. Unfortunately, provided that it is often difficult to foresee the fate of the side branch after main-vessel stenting, we have developed a simplified approach to select cases for elective double stenting, which we propose in Figure 3.

First of all, the clinical relevance of side branches should be assessed in non-left main bifurcations (in distal left main bifurcation, the side branch should always be considered relevant). We consider that side branches $\geq 2.5 \mathrm{~mm}$ diameter and longer than $10 \mathrm{~cm}$ will probably supply $>10 \%$ of myocardium. We propose an arbitrary threshold of $10 \mathrm{~cm}$, which is longer than the value of $73 \mathrm{~mm}$ identified by Kim et al. at CT scan, because angiography has a higher resolution compared to CT and therefore vessels usually look longer. ${ }^{13}$

Once a side branch is judged clinically relevant, the extent of its disease - lesion length - should be taken into account: if longer than $10 \mathrm{~mm}$, then an elective two-stent technique is the preferred approach. Also, the presence of severe calcification on the ostium of a clinically relevant side branch makes us lean towards double stenting. In all other cases, PS will be the preferred option. The choice of the most appropriate two-stent technique goes beyond the aim of this study, and must be individualized according to the anatomical features of the lesion, as well as operator's experience. However, all strategies require a step-by-step approach to reach an optimal final result that favourably impacts the clinical outcome.

\section{Conclusions}

In summary, while PS is appropriate for most cases, in select situations, it is appropriate to start upfront with a double-stenting technique. Based on our experience and data from literature, we provided a simplified approach to bifurcations: we advise a two-stent technique when the side branch is clinically relevant and has significant disease, defined as lesion length $>10 \mathrm{~mm}$ and/or presence of severe calcification. The proposed algorithm is simple and easily reproducible; however, we recognize that the proposed thresholds are arbitrary and require validation in a prospective clinical trial.

\section{Limitations}

We acknowledge that the present study has some shortcomings. Firstly, as with all algorithms, our proposal necessarily represents a compromise between ease of use and ability to capture the complexity of individual situations. Other features not included in this system (such as bifurcation angle, presence of thrombus, lesion length on main vessel, vessel tortuosity) should not be disregarded in appropriate decision making. However, the most important variables seem to be lesion length and calcification on side branch. Secondly, given the retrospective nature of this analysis, our conclusions should be regarded as preliminary and should be validated prospectively in an adequately large cohort of patients. Lastly, intracoronary imaging (IVUS/optical coherence tomography) was not used much in this study, especially in the complicated bifurcation group and the unprotected left main disease. $]$
1. Steigen TK, Maeng M, Wiseth R, et al. Randomized study on simple versus complex stenting of coronary artery bifurcation lesions: The Nordic bifurcation study. Circulation. 2006;114:1955-61.

2. Ferenc $M$, Gick $M$, Kienzle RP, et al. Randomized trial on routine vs. provisional T-stenting in the treatment of de novo coronary bifurcation lesions. Eur Heart J. 2008;29:2859-67.

3. Colombo A, Bramucci E, Saccà S, et al. Randomized study of the crush technique versus provisional side branch stenting in the crush technique versus provisional side-branch stenting in Application of the crushing technique using sirolimus-eluting Application of the crushing technique using
stents) study. Circulation. 2009;119:71-8.

4. Hildick-Smith D, de Belder AJ, Cooter N, et al. Randomized trial of simple versus complex drug-eluting stenting for bifurcation lesions: the British bifurcation coronary study: Old, new, and evolving strategies. Circulation. 2010;121:1235-43.

5. Hildick-Smith D, Behan MW, Lassen JF, et al. The EBC TWO Study (European bifurcation coronary TWO): A randomized comparison of provisional T-stenting versus a systematic 2 stent culotte strategy in large caliber true bifurcations. Circ Cardiovasc Interv. 2016;9:e003643.

6. Chen SL, Santoso T, Zhang JJ, et al. A randomized clinical study comparing double kissing crush with provisional stenting for treatment of coronary bifurcation lesions. J Am Coll Cardiol. 2017:70:2605-17.

7. Moussa ID. Coronary artery bifurcation interventions: The disconnect between randomized clinical trials and patient centered decision-making. Catheter Cardiovasc Inter. 2011;77:537-45.

8. Colombo A, Al-Lamee R. Editorial: Bifurcation lesions: An inside view. Circ Cardiovasc Interv. 2010;3:94-6.

9. Chen SL, Sheiban I, Xu B, et al. Impact of the complexity of bifurcation lesions treated with drug-eluting stents: The DEFINITION study (Definitions and impact of complex bifurcation lesions on clinical outcomes after percutaneous coronary intervention using drug-eluting stents). JACC Cardiovasc Interv. 2014;7:1266-76.

10. Zhang JJ, Ye F, Xu K, et al. Multicentre, randomized comparison of two-stent and provisional stenting techniques in patients with complex coronary bifurcation lesions: The DEFINITION II with complex coronary bifurcation lesio
trial. Eur Heart J. 2020;41:2523-36.

11. Shaw LJ, Berman DS, Picard MH, et al. Comparative definitions for moderate-severe ischemia in stress nuclear, echocardiography, and magnetic resonance imaging. JACC Cardiovasc Imaging. 2014;7:593-604. 
12. Hachamovitch R, Hayes SW, Friedman JD, et al. Comparison of the short-term survival benefit associated with revascularization compared with medical therapy in patients revascularization compared with medical therapy in patient with no prior coronary artery disease undergoing stress myocardial perfusion single photon emission
tomography. Circulation. 2003:107:2900-7.

13. Kim HY, Doh JH, Lim HS, et al. Identification of coronary artery side branch supplying myocardial mass that may benefit from revascularization

14. Wolny R, Pregowski J, Kruk M, et al. Computed tomography angiography versus angiography for guiding percutaneous coronary interventions in bifurcation lesions - A prospective randomized pilot study. I Cardiovasc Comput Tomogr. 2017:11:119-28

15. Koo BK, Lee SP, Lee JH, et al. Assessment of clinical, electrocardiographic, and physiological relevance of diagonal branch in left anterior descending coronary artery bifurcation lesions. JACC Cardiovasc Interv. 2012;5:1126-32.

16. Koo BK, Park KW, Kang HJ, et al. Physiological evaluation of the provisional side-branch intervention strategy for of the provisional side-branch intervention strategy for
bifurcation lesions using fractional flow reserve. Eur Heart J. 2008:29:726-32.

17. Bilge M, Ali S, Alemdar R, et al. First experience with the jailed pressure wire technique in the provisional side branch stenting of coronary bifurcation lesions. Eurolntervention. 2014;10:570-3.

18. Omori $\mathrm{H}$, Kawase $\mathrm{Y}$, Hara $\mathrm{M}$, et al. Feasibility and safety of jailed-pressure wire technique using durable optical fiber pressure wire for intervention of coronary bifurcation lesions. Catheter Cardiovasc Interv. 2019;94:E61-6.

19. Lee $\mathrm{CH}$, Choi SW, Hwang J, et al. 5-year outcomes according to FFR of left circumflex coronary artery after left main crossover stenting. JACC Cardiovasc Interv 2019:12:847-55.

20. Koo BK, Kang HJ, Youn TJ, et al. Physiologic assessment of jailed side branch lesions using fractional flow reserve. J Am Coll Cardiol. 2005:46:633-7.

21. Kumsars I, Narbute I, Thuesen L, et al. Side branch fractiona flow reserve measurements after main vessel stenting: $A$ Nordic-Baltic bifurcation study III substudy. Eurolntervention 2012;7:1155-61.

22. Chen S-L, Ye F, Zhang J-J, et al. Randomized comparison of FFR-guided and angiography-guided provisional stenting of true coronary bifurcation lesions: The DKCRUSH-VI trial (Double kissing crush versus provisional stenting technique for treatment of coronary bifurcation lesions VI). JACC CardiovasC Interv. 2015;8:536-46.

23. Kang SJ, Mintz GS, Kim WJ, et al. Preintervention angiographic and intravascular ultrasound predictors for side branch compromise after a single-stent crossover technique. Am Cardiol. 2011;107:1787-93.

24. Kang SJ, Kim WJ, Lee JY, et al. Hemodynamic impact of changes in bifurcation geometry after single-stent cross-over technique assessed by intravascular ultrasound and fractional flow reserve. Catheter Cardiovasc Interv. 2013;82:1075-82.

25. Hahn JY Chun WJ, Kim JH, et al. Predictors and outcomes of side branch occlusion after main vessel stenting in coronary bifurcation lesions: Results from the COBIS II registry (Coronary bifurcation stenting). J Am Coll Cardiol. 2013;62:1654-9.

26. Dou K, Zhang D, Xu B, et al. An angiographic tool for risk prediction of side branch occlusion in coronary bifurcation intervention: The RESOLVE score system (Risk prediction of side branch occlusion in coronary bifurcation intervention). JACC Cardiovasc Interv. 2015;8:39-46.

27. Di Gioia G, Sonck J, Ferenc M, et al. Clinical outcomes following coronary bifurcation $\mathrm{PCl}$ techniques: A systematic review and network meta-analysis comprising 5,711 patients. JACC Cardiovasc Interv. 2020;13:1432-44.

28. Dzavik V, Kharbanda R, Ivanov J, et al. Predictors of long-term outcome after crush stenting of coronary bifurcation lesions: Importance of the bifurcation angle. Am Heart J. 2006;152:762-9

29. Goto Y Kawasaki T, Koga $N$ et al. Plaque distribution patterns in left main trunk bifurcations: Prediction of branch vessel compromise by multidetector row computed topography compromise by multidetector row computed topography after percutan

30. Kim MC, Ahn Y, Sim DS, et al. Impact of calcified bifurcation lesions in patients undergoing percutaneous coronary intervention using drug-eluting stents: Results from the Coronary blfurcation stent (COBIS) II registry. Eurolntervention. 2017; 13:338-44.

31. Bai J, Yue Y, Feng HQ, et al. Impact of main vessel calcification on procedural and clinical outcomes of bifurcation lesion undergoing provisional single-stenting intervention: $\mathrm{A}$ multicenter, prospective, observational study. I Geriatr Cardiol. 2019;16:156-63.

32. Allali A, Abdel-Wahab M, Traboulsi $\mathrm{H}$, et al. Impact of lesion preparation technique on side branch compromise in calcified coronary bifurcations: A subgroup analysis of the coronary bifurcations: A subgroup analysis of the

33. Barman N, Okamoto N, Ueda H, et al. Predictors of side branch compromise in calcified bifurcation lesions treated with orbital atherectomy. Catheter Cardiovasc Interv. 2019;94:45-52.

34. Sturm R, Armstrong EJ, Benhuri B, et al. Orbital atherectomy for treatment of severely calcified coronary artery bifurcation lesions: A multicenter analysis. Cardiovasc Revascularization Med. 2021;26:34-8

35. Ford TJ, McCartney P, Corcoran D, et al. Single- versus 2-stent strategies for coronary bifurcation lesions: A systematic review and meta-analysis of randomized trials with long-term follow-up. J Am Heart Assoc. 2018;7:e008730 